\title{
Entropy of Extremal Black Holes in Two Dimensions
}

\author{
Alok Kumar and Koushik Ray \\ Institute of Physics, \\ Bhubaneswar 751 005, INDIA \\ email: kumar, koushik@iopb.ernet.in
}

July 24, 2018

\begin{abstract}
Entropy for two dimensional extremal black holes is computed explicitly in a finite-space formulation of the black hole thermodynamics and is shown to be zero locally. Our results are in conformity with the recent one by Hawking et al in four dimensions.
\end{abstract}


It is known that black holes have temperature and entropy and are therefore amenable to a thermodynamic description. For an asymptotic observer, the temperature of the black hole is given by the surface gravity on the horizon and the entropy is one quarter the horizon area. It was shown by Gibbons and Hawking[1] that the entropy of the black hole coincides with the value of the Euclideanized action at infinity. The laws of black hole thermodynamics were also formulated [2, 3]. It was also thought that for charged black hole, e.g. Reissner-Nordström solution, one had a non-vanishing entropy even in the extremal limit (viz. charge $\rightarrow$ mass) although the temperature tends to zero. This is essentially due to the fact that extreme black holes have non-zero area. As a result, although the extremal limit was known to be unattainable due to the cosmic censorship [4], there was no purely thermodynamic way to establish this. In thermodynamics, unattainability of absolute zero by adiabatic processes follows from Nernst's postulate of the vanishing of entropy in this limit [5]. It has been realized only recently[[6, 7] that extreme black holes in four space-time dimensions do have zero entropy as measured at infinity.

Recently we have studied the thermodynamics of several types of black hole solutions in two dimensions in a finite-space formulation [8] devised by Gibbons and Perry[9]. It was also commented that our results applied only to non-extremal black holes. The extremal black holes needed separate treatment.

In this paper we apply the method of [8] to the extremal black holes in 2D and show that the entropy in these cases is also zero which corroborates the results of [6]. However in the present formulation the local entropy itself turns out to be zero. In this regard the extreme black holes are thermodynamically similar to the linear dilaton vacuum solutions. We also compute the energy of the extreme black holes from thermodynamic considerations and reproduce the ADM mass. We deal with two extremal black hole solutions. One of these is an asymptotically flat solution for 
the heterotic string [10]. The other black hole considered is an asymptotically anti-de Sitter one ensuing from a charged version of the Jackiw-Teitelboim theory discussed by Lowe and Strominger [11].

Finite-space formulation of thermodynamics is discussed for the non-extreme cases in [9] and [8]. It was found that the entropy turns out to be non-zero on setting charge equal to mass in the entropy for the non-extremal ones. However, note that, in this scheme the metric is written in a particular gauge, viz.

$$
d s^{2}=g_{t t} d t^{2}+d \rho^{2} .
$$

This metric is singular in the limit charge $\rightarrow$ mass. Actually the coordinate transformations from Schwarzschild to these coordinates itself is invalid in this limit. Therefore to discuss the thermodynamics of extremal black holes we shall change the coordinate after setting charge equal to mass. In the transformed coordinates, $(\mathrm{t}, \rho)$, for the extreme cases the horizon is at $\rho=-\infty$ and the surface gravity at the horizon turns out to be zero. We shall treat the temperature, i.e. the inverse periodicity of the Euclidean time at the horizon, as arbitrary till the end. Thermodynamic calculations are then the same as in [9, 8].

Let us now review the thermodynamic definitions in the present scheme. We begin with the definition of free energy:

$$
\mathcal{F}=\frac{\mathcal{I}}{\beta},
$$

where $\mathcal{I}$ is the Euclideanized action evaluated for the metric of the space-time under consideration and $\beta$ is the inverse temperature. Therefore we will first have to evaluate the actions for the cases at hand. Since we are concerned with a finite-space formulation of thermodynamics, we have to evaluate the action with the boundary contribution on a spacelike slice in the above mentioned coordinates. This is feasible 
since the metric admits a Killing vector $k^{a}=\left(\frac{\partial}{\partial t}\right)^{a}$. The inverse proper periodicity of the Euclideanized time at a fixed value of the spatial coordinate is interpreted as the local temperature $T_{w}=\beta^{-1}=T_{c} / \sqrt{g_{t t}}$, where $T_{c}$ is the inverse periodicity at the horizon. Note that since $T_{c}=0$ in the cases at hand, the thermodynamic quantities are to be interpreted as a limiting value. For example,

$$
\mathcal{F}=\lim _{T_{c} \rightarrow 0} \frac{\mathcal{I}}{\beta} .
$$

We will, for convenience of writing, not mention the limits explicitly in the following. The electric charge, $Q$, and the dilaton charge

$$
\mathcal{D}=\int_{\Sigma} j
$$

define the other thermodynamic potentials. Here $j_{a} \equiv \epsilon_{a}^{b} \nabla_{b} e^{-2 \phi}$ is a conserved current and $\Sigma$ is a spacelike hypersuface. We will therefore treat $\mathcal{F}$ as a function of the thermodynamic quantities $T_{w}, Q$ and $\mathcal{D}$. Then by the first law of thermodynamics, one can define the entropy $S$ and the dilaton potential $\psi$ as:

$$
S=-\left[\frac{\partial \mathcal{F}}{\partial T_{w}}\right]_{\mathcal{D}}, \quad \psi=-\left[\frac{\partial \mathcal{F}}{\partial \mathcal{D}}\right]_{T_{w}} .
$$

The quantity $\mathcal{E}=\mathcal{F}+S T_{w}$, corresponds to the mass of the black hole, provided it exsists, as the limiting value of the difference between the energies for the black hole and its asymptotic background solution at spatial infinity. An alternative thermodynamic scheme is developed in 12 by subtracting infinities in the action. The present formulation sweeps these infinities under the carpet as a divergent contribution to the chemical potential $\psi$.

We now start with the discussions of the charged 2D black hole in the heterotic string theory [10]. These emerge as the solutions to the action:

$$
\mathcal{I}=-\int_{\mathcal{M}} \sqrt{g} e^{-2 \phi}\left[R+4(\nabla \phi)^{2}+\lambda^{2}-\frac{1}{2} F^{2}\right]-2 \int_{\partial \mathcal{M}} e^{-2 \phi} K
$$


where $K$ is the trace of the second fundamental form and $\partial \mathcal{M}$ is the boundary of $\mathcal{M}$. A black hole solution of this theory can be written as:

$$
\begin{aligned}
d s^{2} & =-\mathcal{G}(r) d t^{2}+\frac{1}{\mathcal{G}(r)} d r^{2} \\
\mathcal{G}(r) & =1-2 m e^{-\lambda r}+q^{2} e^{-2 \lambda r} \\
e^{-2 \phi} & =e^{-2 \phi_{0}} e^{\lambda r} \\
A_{0} & =\sqrt{2} q e^{-\lambda r} .
\end{aligned}
$$

Thermodynamics of this black hole has been discussed in [9] for the non-extreme case. The solution can be written in the form (11) as:

$$
\begin{aligned}
d s^{2} & =-\frac{\left(1-\frac{q^{2}}{m^{2}}\right) \sinh ^{2} \lambda \rho}{\left(1+\sqrt{1-\frac{q^{2}}{m^{2}}} \cosh \lambda \rho\right)^{2}} d t^{2}+d \rho^{2}, \\
\phi & =\phi_{0}-\frac{1}{2} \log \left[m+\sqrt{m^{2}-q^{2}} \cosh \lambda \rho\right], \\
A_{0} & =\frac{\sqrt{2} q}{m+\sqrt{m^{2}-q^{2}} \cosh \lambda \rho},
\end{aligned}
$$

and the asymptotic entropy for the black hole is

$$
S=\frac{\pi m}{\lambda}\left[1+\left(1-\frac{q^{2}}{m^{2}}\right)^{1 / 2}\right],
$$

which is $\frac{\pi m}{\lambda}$ in the extremal limit $\frac{q^{2}}{m^{2}} \rightarrow 1$. Another prescription to get non-zero asymptotic entropy in the extremal limit is also suggested in [12]. However, note that, the transformation between the two coordinates $r$ and $\rho$, viz.

$$
r=\frac{1}{\lambda} \log \left(m+\sqrt{m^{2}-q^{2}} \cosh \lambda \rho\right)
$$

is valid only for $q<m$ and the metric (11) is not defined in the extremal limit. For a consistent description, therefore, we have to start from (17) with $q=m$ and then transform the coordinates:

$$
r=\frac{1}{\lambda} \log \left(m+e^{\lambda\left(\rho-\rho_{0}\right)}\right),
$$


where $\rho_{0}$ is a choice of integration constant. Starting anew with the coordinate transformation is in conformity with [6] where it is maintained that the extremal and non-extremal black holes are different objects. Note that, the extremal black hole has a different topology than the non-extreme one. The horizon is now situated at $\rho=-\infty$ unlike the non-extreme case, where horizon was at $\rho=0$. Now both the black hole and the asymptotic space are described using the same chart. This can be seen from (16). By setting $\rho_{0}=0, \rho$ and $r$ in fact coincide for $m=0$. This is of import in the calculation of the mass of the black hole. The transformed extremal solution takes the form,

$$
\begin{aligned}
d s^{2} & =-\mathcal{G}(\rho) d t^{2}+d \rho^{2} \\
\mathcal{G}(\rho) & =\frac{e^{2 \lambda\left(\rho-\rho_{0}\right)}}{\left(m+e^{\lambda\left(\rho-\rho_{0}\right)}\right)^{2}} \\
\phi & =\phi_{0}-\frac{1}{2} \log \left[\left(m+e^{\lambda\left(\rho-\rho_{0}\right)}\right)\right] \\
A_{0} & =\frac{\sqrt{2} m}{m+e^{\lambda\left(\rho-\rho_{0}\right)}} .
\end{aligned}
$$

We have kept the constant $\rho_{0}$ in the above expressions. We will however see later on that thermodynamics is independent of $\rho_{0}$. The extreme black hole also asymptotes to the flat space with linear dilaton

$$
\begin{aligned}
d s^{2} & =-d t^{2}+d \rho^{2} \\
\phi & =\phi_{0}-\frac{1}{2} \lambda\left(\rho-\rho_{0}\right) .
\end{aligned}
$$

We now evaluate the Euclidean action for the black hole (77)-(10). It takes the form

$$
\mathcal{I}=-\int_{\partial \mathcal{M}} \sqrt{\frac{1}{g_{11}}} e^{-2 \phi}\left(\frac{1}{2} \frac{\partial_{1} g_{00}}{g_{00}}-2 \partial_{1} \phi\right)
$$

The dilaton charge is found to be

$$
\mathcal{D}=e^{-2 \phi_{0}}\left(m+e^{x}\right), \quad \text { where } \quad x=\lambda\left(\rho-\rho_{0}\right) .
$$


The free energy of this black hole is then obtained by dividing the action by the local temperature and takes the form

$$
\mathcal{F}=-2 \lambda \mathcal{D}
$$

We note that the free energy is dependent on only one thermodynamic quantity, namely the dilaton charge. Hence the entropy as defined in (5) is identically zero. It also implies that the thermal energy $\mathcal{E}=\mathcal{F}=-2 \lambda \mathcal{D}$. Calculating the free energy in the similar way for the flat space linear dilaton vacuum one finds

$$
\begin{aligned}
\mathcal{D}_{\mathrm{fs}} & =e^{-2 \phi_{0}} e^{x} \\
\mathcal{F} & =-2 \lambda \mathcal{D}_{\mathrm{fs}} \\
S & =0 \\
\mathcal{E}_{\mathrm{fs}} & =-2 \lambda \mathcal{D}_{\mathrm{fs}}
\end{aligned}
$$

Hence the mass of the black hole can be obtained as the asymptotic value of the energy differene of the two space-times as

$$
M=\lim _{x \rightarrow \infty}\left(\mathcal{E}-\mathcal{E}_{\mathrm{fs}}\right)=m e^{-2 \phi_{0}}
$$

which is in fact the ADM mass of the black hole. Note that unlike the non-extremal cases [9, 8], there is no change of variable involved in calculating $M$ due to their description by the same charts in this case. Interestingly, we also see the similarity in the expressions for free energies of the black hole and the linear dilaton vacuum solutions.

Now let us turn to the study of the black hole obtained in [11] by introducing gauge fields through the dimensional compactification of a three dimensional string effective action. The thermodynamics for the non-extremal case was discussed in [8]. The $2 \mathrm{D}$ action in this case has the form,

$$
\mathcal{I}=-\int_{\mathcal{M}_{2}} \sqrt{g} e^{-2 \Phi}\left[R+2 \lambda^{2}-\frac{1}{4} e^{-4 \Phi} F^{2}\right]-2 \int_{\partial \mathcal{M}} e^{-2 \Phi} K,
$$


where now $\Phi$ is a scalar field coming from the compactification and plays the role of dilaton for the 2D action. Action (31) describes the Jackiw-Teitelboim theory with a gauge field. This theory possess the black hole solution

$$
\begin{aligned}
d s^{2} & =-\left(\lambda^{2} r^{2}-m+\frac{J^{2}}{4 r^{2}}\right) d t^{2}+\left(\lambda^{2} r^{2}-m+\frac{J^{2}}{4 r^{2}}\right)^{-1} d r^{2}, \\
A_{0} & =-\frac{J}{2 r^{2}} \\
e^{-2 \Phi} & =r .
\end{aligned}
$$

The parameter $J$ in this solution gives charge to this black hole. The metric has a curvature singularity at $r=0$ for nonvanishing $J$ as is seen from the Ricci scalar

$$
R=-2 \lambda^{2}-\frac{3 J^{2}}{2 r^{4}}
$$

It goes asymptotically, $r \rightarrow \infty$, to the anti-de Sitter Space-time. Now we study the thermodynamics of these black hole solutions in the extremal case. The use of the equations of motion implies the following value of the classical action 8 :

$$
\mathcal{I}=-\int_{\partial \mathcal{M}}\left[n^{a} F_{a b} A^{b} e^{-6 \Phi}+2 K e^{-2 \Phi}\right]
$$

We now rewrite the solution (32)-(34) in the extremal case, $m^{2}=\lambda^{2} J^{2}$, in the form (11):

$$
r^{2}=\frac{m}{2 \lambda^{2}}\left[1+e^{2 \lambda \rho}\right] .
$$

We find

$$
d s^{2}=-\mathcal{G}(\rho) d t^{2}+d \rho^{2},
$$

where

$$
\mathcal{G}(\rho)=\left(\frac{m}{2}\right) \frac{e^{4 \lambda \rho}}{1+e^{2 \lambda \rho}} .
$$


$A_{0}$ and $e^{-2 \Phi}$ are still given by (33)-(34) with $r$ replaced from (37). In these coordinates the horizon is again at $\rho=-\infty$. The free energy is obtained by the evaluation of (36). We note however that there is an ambiguity in the evaluation of (36) due to the freedom of a constant shift in the gauge potential: $A_{a} \rightarrow A_{a}+$ const., in the equations of motion. Constant shifts on gauge potentials have been applied earlier [13, 1]. This shift was also needed in [8] for consistency of the present method of computations with the Noether's charge prescription [14].

The dilaton charge $\mathcal{D}$ is now given by

$$
\mathcal{D}=\left[\frac{m}{2 \lambda^{2}}\left(1+e^{2 x}\right)\right]^{1 / 2}, \quad \text { where } \quad x=\lambda \rho .
$$

Evaluating (36), with a shift in the gauge potential $A_{\mu} \rightarrow A_{\mu}(\rho)-A_{\mu}(\rho=-\infty)$ the free energy once again takes the form

$$
\mathcal{F}=-2 \lambda \mathcal{D}
$$

Hence the entropy is zero again. The thermal energy of the black hole is to be computed with reference to the AdS linear dilaton vacuum defined by

$$
\begin{aligned}
d s^{2} & =-\frac{m}{2} e^{2 \lambda \rho} d t^{2}+d \rho^{2}, \\
\Phi & =-\frac{1}{2} \log \sqrt{\frac{m}{2 \lambda^{2}}}-\frac{1}{2} \lambda \rho .
\end{aligned}
$$

The free energy becomes $\mathcal{F}_{A d S}=-2 \lambda \mathcal{D}_{A d S}$, which implies $S_{A d S}=0$ and $\mathcal{E}_{\text {AdS }}=$ $-2 \lambda \mathcal{D}$. Then defining $M \equiv \mathcal{E}-\mathcal{E}_{A d S}$ we obtain

$$
M=\left(\frac{m}{2}\right)^{1 / 2} e^{-x}
$$

This gives the correct ADM mass of the black hole, $\frac{m}{2}$, by taking into account the redshift factor.

To conclude, we have shown that the entropy for the extremal black holes vanishes in two dimensions. We in fact found that the entropy vanishes locally as could 
be expected from the vanishing of the local temperature. It will be intersting to understand these results from the microscopic point of view. Also, two dimensional heterotic string theory have another type of charged black hole solution[15]. It will be interesting to investigate the thermodynamics of these solutions. 


\section{References}

[1] G. Gibbons and S. Hawking, Phy. Rev. D15, 2752 (1977).

[2] R. M. Wald, General Relativity, University of Chicago Press (1984).

[3] C. Lousto, Nucl. Phys. B410, 155 (1993).

[4] I. Novikov and V. Frolov, Physics of Black Holes, Kluwer Academic, Dordrecht (1989).

[5] H. B. Callen, Thermodynamics, J Wiley and Sons Inc. (1960).

[6] S. Hawking, G. Horowitz and S. Ross, Cambridge University Preprint, DAMTP/R 94-26; gr-qc/9409013.

[7] C. Teitelboim, hep-th/9410103.

[8] A. Kumar and K. Ray, IP/BBSR/94-54, hep-th/9410068.

[9] G. Gibbons and M. Perry, Int. J. Mod. Phys.D1, 335 (1992).

[10] M.D.Mc.Guian, C.R.Nappi and S.A.Yost, Nucl. Phys. B375, 421 (1992).

[11] D. Lowe and A. Strominger, Phys. Rev. Lett.73,1468 (1994).

[12] C. R. Nappi and A. Pasquinucci, Mod. Phys. Lett. A7, 3337 (1992).

[13] R. Kallosh, T. Ortin and A. Peet, Phy. Rev. D47, 5400 (1993).

[14] R. M. Wald, Phy. Rev. D48, 3427 (1993); V. Iyer and R. M. Wald, gr-qc/9403028.

[15] A. Giveon, E. Rabinovici and A. A. Tseytlin, Nucl. Phys. B409, 339 (1993). 